\title{
Sonolência excessiva diurna e depressão: causas, implicações clínicas e manejo terapêutico
}

\author{
Excessive daytime sleepiness and depression: causes, clinical implications, \\ and therapeutic management
}

\author{
Sarah Laxhmi Chellappa* \\ * Médica. Centre for Chronobiology, Psychiatric Hospital of the University of Basel, Basel, \\ Switzerland. \\ Endereço para correspondência
}

\begin{abstract}
RESUMO
A sonolência excessiva diurna (SED) é frequentemente associada à depressão, e as possíveis relações entre as duas afecções são numerosas. A SED pode ocorrer devido a insônia ou hiperssonia. A sintomatologia da depressão inclui, notadamente, a insônia e a consequente SED, que podem ser, em alguns casos, sintomas residuais após a resposta ao tratamento antidepressivo. Paralelamente, a insônia e a sonolência diurna podem, inclusive, ser efeitos colaterais de curta ou longa duração do manejo terapêutico antidepressivo. Independente de a SED ser um sintoma de um quadro depressivo atual, sintoma residual de depressão prévia ou efeito colateral de medicação antidepressiva, faz-se necessária uma adequada avaliação clínica da SED na depressão. A fim de discorrer sobre as atuais evidências das investigações da SED na depressão, foi feito um levantamento da literatura médica nos bancos de dados ISI, MEDLINE e SciELO, compreendendo-se o período de 1990 a 2007 . Apesar de os mecanismos responsáveis pela relação entre a sonolência diurna e a depressão serem complexos e entrelaçados, a avaliação compreensiva desse transtorno do sono desempenha um papel fundamental na predição de respostas ao manejo terapêutico, recaídas e modelos etiológicos da depressão.
\end{abstract}

Descritores: Sono, distúrbios do sono por sonolência excessiva, transtornos do início e da manutenção do sono, transtorno depressivo.

\footnotetext{
ABSTRACT

Excessive daytime sleepiness (EDS) is often related to depression, and there are several possible relationships between them. EDS can usually occur due to either insomnia or hypersomnia. The symptomatology of depression prominently includes insomnia and the resultant EDS, which may, in such cases, be residual symptoms as a response to antidepressant treatment. Furthermore, insomnia and daytime sleepiness may be short- or long-term side effects of antidepressant treatment as well. The clinical assessment of depression should adequately address whether EDS is a symptom of current depression, a prior depression residual symptom, or a side effect of antidepressant medication. With the purpose of showing current evidence about the investigations of EDS related to depression, we conducted a review of the literature using the following databases: ISI, MEDLINE and SciELO from 1990 to 2007. Although the underlying mechanisms of EDS in depression are complex and intertwined, the comprehensive assessment of this sleep disorder plays a key role in areas related to the prediction of responses to treatment, relapses, and etiological models in depression.
} 
Keywords: Sleep, disorders of excessive somnolence, sleep initiation and maintenance disorders, depressive disorder.

\section{NTRODUÇÃO}

Aproximadamente $80 \%$ dos pacientes com transtorno depressivo apresentam alterações nos padrões do sono, tanto de uma perspectiva qualitativa como quantitativa. Deles, a maioria apresenta insônia, incluindo suas queixas específicas despertares noturnos frequentes, sono não restaurador, redução do sono total e sonhos perturbadores provocando hiperssonolência diurna ${ }^{1-7}$. Em aproximadamente 10 a $20 \%$ dos pacientes com depressão unipolar grave, o sono é anormalmente mais longo - casos de hiperssonia - e concomitante à sonolência excessiva diurna (SED), principalmente em adultos jovens ${ }^{8}$. Por sua vez, na depressão bipolar, a insônia e a SED podem estar presentes ${ }^{9}$. Os pacientes com doença afetiva sazonal e com depressão atípica também apresentam quadros de hiperssonia associados à depressão ${ }^{10}$. Recentemente preconizadas pelo American Academy of Sleep Medicine ${ }^{6}$, as alterações do sono, como a insônia e a sonolência excessiva (SE), podem ser comórbidas à depressão, principalmente quando essas queixas persistem por mais de 6 meses $^{11}$. Como referido previamente, um número expressivo de casos de SED é decorrente da insônia e podem tanto ser sintomas ou indicadores da depressão, quanto, inclusive, efeitos colaterais do tratamento antidepressivo ${ }^{12}$.

A SED e o aumento da vigília noturna estão associados à depressão de diversas formas, e essas relações devem ser identificadas e compreendidas, contribuindo assim para o diagnóstico daquele transtorno, a elaboração de estratégias de tratamento e a identificação de indivíduos vulneráveis ao desenvolvimento da depressão ${ }^{13}$.

\section{MÉTODO}

O presente estudo consiste em uma revisão sistemática da relação da SED com o transtorno depressivo. O levantamento bibliográfico foi realizado nos bancos de dados indexados no ISI, MEDLINE e SciELO mediante uma busca de artigos publicados de 1990 a 2007 em inglês, espanhol e português. Para refinar essa busca, foram utilizados apenas os seguintes descritores cadastrados nos bancos de dados de descritores em Ciências da Saúde: sono; transtornos do sono; transtornos do início e da manutenção do sono; transtornos do sono por SE; transtorno depressivo; ideação suicida; Neurobiologia; ritmos circadianos; saúde mental; Epidemiologia. Desta forma, foram incluídos artigos originais (29) e de revisão sistemática e não sistemática (22) e excluídos relatos de caso e cartas ao editor.

\section{DISCUSSÃO}

\section{Bases fisiológicas da SED}

Do ponto de vista conceitual, a SED pode ser considerada o resultado de três fatores: sonolência fisiológica, manifesta e introspectiva. A sonolência fisiológica é o resultado da necessidade biológica de sono, sendo desencadeada pela complexa interação da necessidade homeostática do sono, do componente circadiano e da inércia do sono ${ }^{14,15}$.

A necessidade de sono é a quantidade de sono requerida para manter a alerta durante a vigília. Para tanto, o processo S (definido sucintamente como a pressão do sono) aumenta ao longo do dia e atinge o máximo à noite, levando ao sono. Na depressão, esse aumento do processo $\mathrm{S}$ é deficiente durante a vigília devido à diminuição do sono de ondas lentas. Como consequência, há um maior débito do sono e mais SED ${ }^{16,17}$. O componente circadiano é responsável pela sincronização do ciclo sono-vigília ao ciclo claro-escuro, mediado pelo núcleo supraquiasmático (denominado "relógio biológico"). Na depressão, o controle dos ritmos circadianos, como sono REM (rapid eye movement), 
temperatura corporal e secreção de cortisol, é desregulado em relação ao ciclo de sono-vigília ${ }^{18}$. Consequentemente, os pacientes dormem comparativamente mais tarde em relação ao próprio relógio biológico, resultando na insônia e na SED. A inércia do sono dura entre 5 e 30 minutos em indivíduos sem alterações do sono, mas ela é maior em pacientes com privação do sono ou com alterações do sono ${ }^{14,15}$, como em pacientes depressivos, evidenciando-se na dificuldade de despertar.

A sonolência manifesta corresponde ao efeito comportamental da necessidade fisiológica modulada pela motivação individual e por fatores ambientais. Em relação a esse componente, são observados na depressão redução do desempenho e da alerta do indivíduo sonolento, episódios de sono involuntário apesar das tentativas volitivas de manter-se acordado e menor desempenho em testes cognitivos e comportamentais ${ }^{8,19}$.

A sonolência introspectiva é a habilidade individual de caracterizar o estado interno pessoal. Em indivíduos normais, há uma adequada percepção da sonolência após privação do sono aguda. Todavia, em casos de privação crônica decorrentes de alterações clínicas como a depressão, a percepção da sonolência é menor e os pacientes tendem a desvalorizar as queixas de sonolência ${ }^{9,20}$. Esta incapacidade de identificar a sonolência na depressão pode acarretar a demora do paciente em procurar tratamento médico e/ou uma inadequada investigação na anamnese clínica ${ }^{21}$. Desta forma, a compreensão e o manejo adequado dessa alteração do sono são fundamentais na avaliação clínica do paciente depressivo.

Insônia, hiperssonia e depressão

O transtorno do sono mais comumente encontrado no transtorno depressivo é a insônia, caracterizada, principalmente, pelo sono inadequado e/ou não restaurador, com consequências diurnas como irritabilidade, deficit de concentração e de memória e SED ${ }^{1,2}$. A insônia pode atuar como importante indicador na avaliação do subsequente desenvolvimento da depressão ${ }^{12,22}$ e pode, inclusive, indicar tanto um novo episódio depressivo como corroborar o seu surgimento.

A idade dos pacientes constitui um importante fator de avaliação, pois, com o avançar da idade, há outras alterações de sono que incluem maior fragmentação do sono noturno e despertar mais precoce $^{23}$. Em relação ao sexo, são observadas nas mulheres mais queixas de insônia e de hiperssonia (proporção 2:1), apesar de haver equivalência entre os sexos após os 65 anos ${ }^{24}$. Fatores psicossociais como menor nível educacional, presença de comorbidades - hipertensão, artrite reumatoide e diabetes -, entre outros ${ }^{1,2,9}$, podem estar relacionados a alterações do sono. Apesar de a insônia ser mais prevalente, a SE é um transtorno do sono que faz o paciente buscar tratamento médico ${ }^{8}$. Em 10 a 20\% dos casos, há excesso de sono (hiperssonia) sob a forma de episódios de sono noturno prolongado e aumento do sono diurno ${ }^{10}$, principalmente na depressão grave ou na depressão atípica, com predominância de queixas como aumento de peso, irritabilidade e SE. Contudo, é fundamental a investigação da apneia ou hipopneia obstrutiva do sono nos pacientes, levando em consideração que a relação entre estas e o transtorno depressivo está bem estabelecida, principalmente em mulheres de meia-idade. Em decorrência dessa associação, as síndromes referidas acarretam um sono entrecortado cuja principal consequência é a SED ${ }^{9}$.

\section{SED e depressão}

A SED é considerada um dos principais sintomas do quadro depressivo, podendo decorrer da insônia ou da hiperssonia. Diversos estudos epidemiológicos têm observado uma marcante relação entre esse sintoma e a depressão moderada a grave $23,25,26$. Em um estudo prévio, foi observada uma associação significativa entre a SED e a ideação suicida ${ }^{9}$. Uma possível explicação é a de que pacientes depressivos graves tendem a classificar (ranking) a sonolência como sendo um dos sintomas mais graves do próprio quadro clínico em conjunção com outros sintomas mais clássicos da depressão, como humor melancólico e apatia ${ }^{25}$. No mesmo estudo ${ }^{9}$, pacientes com altos níveis de SE apresentavam uma maior duração do transtorno depressivo em comparação com pacientes com 
pouca sonolência. Essa causalidade pode ser atribuída à relação da SED com quadros depressivos graves $^{27}$. Contudo, a sonolência pode ser decorrente dos seguintes fatores: sintoma residual de depressão prévia; transtorno depressivo bipolar; concomitância de apneia ou hipopneia obstrutiva do sono, principalmente em pacientes na faixa etária de 40 a $55 \operatorname{anos}^{1,8,9}$.

\title{
Diagnóstico de SED na depressão
}

As queixas de sonolência são definidas como semelhantes às sensações de cansaço, fadiga, baixa motivação, atenção/concentração reduzidas ou pouca energia para execução de tarefas ${ }^{28}$. A identificação da SED pode ser consideravelmente difícil, tendo em vista os múltiplos termos utilizados para descrevê-la e/ou pela desvalorização da gravidade desse sintoma pelo paciente. Para muitos pacientes, a identificação da própria sonolência é um desafio devido à dificuldade em definir seu estado fisiológico interno. Além disso, estratégias compensatórias como estender o tempo total de sono para prevenir a sonolência durante a vigília ${ }^{8,10,21}$ podem mascarar a sonolência. Contudo, a alta prevalência de transtornos do sono e sua relação com quadros depressivos graves tornam fundamental uma melhor caracterização dessas queixas na avaliação clínica. Após a identificação das queixas do sono alterado na entrevista inicial, é recomendada a estimativa de horários de início e despertar do sono a fim de determinar a duração total de sono. Para tanto, diários do sono e escalas de sonolência podem auxiliar sobremaneiramente $e^{27,29}$.

As medidas subjetivas como a Escala de Sonolência de Stanford, a Escala de Sonolência de Karolinska e a Escala de Sonolência de Epworth ${ }^{30-32}$ podem ser utilizadas para avaliar a sensação de SE em um dado momento ou em situações diárias e consistem em questionários padronizados respondidos pelo próprio paciente.

Alguns problemas podem estar relacionados às escalas, como, por exemplo, a ambiguidade de algumas situações apresentadas e/ou variações diurnas do grau de SE. Considerando estes aspectos, medidas objetivas de investigação da SE, como a polissonografia (PSG) e o Teste das Latências Múltiplas do Sono (TLMS), podem ser utilizados na avaliação objetiva da SE ${ }^{1,33}$. Simultaneamente, os testes de desempenho e de vigilância detectam a habilidade de sustentar a atenção e a eficiência cognitiva, que tendem a diminuir na privação do sono ${ }^{34}$.

A PSG permite o registro de múltiplas variáveis fisiológicas durante uma noite de sono e constitui uma ferramenta essencial para a avaliação do padrão do sono noturno e o diagnóstico dos transtornos do sono. Deve ser indicada aos pacientes com queixa de SE, exceto àqueles com SE claramente associada à privação de sono ou ao uso de medicamentos ${ }^{10,35}$. Por sua vez, o TLMS é considerado o método de escolha para a avaliação objetiva e o acompanhamento da SED, quantificando esse sintoma e possibilitando a identificação do sono $\operatorname{REM}^{36}$.

Dentre os principais diagnósticos diferenciais, alguns se destacam de forma mais acentuada:

\begin{abstract}
- Apneia e/ ou hipopneia obstrutiva do sono: atinge de 2 a $4 \%$ da população, principalmente homens acima de 40 anos e acima do peso, definindo-se como episódios recorrentes de obstrução parcial (hipopneias) ou total (apneias) da via aérea superior durante o sono. O diagnóstico é definido com base em critérios clínicos e polissonográficos: 1) SED inexplicável por outras causas; 2) dois ou mais dos seguintes sintomas e sinais, não explicados por outras condições: asfixia ou respiração difícil durante o sono, despertar noturno recorrente, sensação de sono não restaurador, fadiga diurna e dificuldade de concentração; 3) monitorização durante a noite inteira, com cinco ou mais eventos respiratórios obstrutivos (apneias e/ou hipopneias) por hora de sono ${ }^{6,37}$;
\end{abstract}

- Narcolepsia: doença rara (1/1.000 indivíduos) com maior pico de incidência entre os 18 e 25 $a_{0}{ }^{5}$. Classicamente, pode apresentar-se com os seguintes sinais e sintomas: SE, cataplexia (perda de tônus muscular), paralisia do sono e sono fragmentado. Pode ser monossintomática, apresentando-se somente com SE, ou oligossintomática, com SE e cataplexia. O grau de SE é variável, e os ataques de cataplexia podem envolver diversos grupos musculares, sendo desencadeados por emoções, principalmente o riso. Na polissonografia, há latências para o início do sono e para o sono REM diminuídas, aumento da quantidade de sono REM e despertares frequentes ${ }^{10}$; 


\begin{abstract}
- Síndrome das pernas inquietas (SPI)/ Movimentos periódicos de membros (MPM): OS MPM incidem frequentemente após os 30 anos, com prevalência de $5 \%$. Dos pacientes com SPI, $80 \%$ apresentam MPM, mas a maioria das pessoas que têm MPM não necessariamente tem SPI ${ }^{11}$. Os critérios diagnósticos para a MPM incluem queixa de insônia ou SE; movimentos de membros estereotipados com extensão do hálux, flexão de perna sobre coxa e desta sobre o quadril; polissonografia com cinco ou mais episódios de MPM por hora de sono. Os critérios para diagnóstico da SPI são clínicos, sendo referida uma sensação desagradável nas pernas, levando à necessidade de movimentá-las, que piora com o repouso ou sono e melhora com a movimentação ${ }^{38}$;
\end{abstract}

- Drogas e medicações: a SED pode se dever ao uso de inúmeras medicações como hipnóticos, anticonvulsivantes, antidepressivos, anti-histamínicos, lítio, antipsicóticos, antiparkinsonianos, drogas cardiovasculares, entre outras ${ }^{39}$.

\title{
Tratamento da SED na depressão
}

Existem diversos tratamentos de opção em potencial específicos para o tipo de sonolência associada à depressão. O esquema terapêutico mais adotado em pacientes com depressão grave e com queixas de insônia e SED é a monoterapia com um tipo de antidepressivo. A escolha desse esquema de tratamento parte do pressuposto de que a melhora do quadro depressivo é acompanhada de subsequentes queixas de alterações do sono. Dentre o leque de medicações antidepressivas utilizadas nestes casos, destacam-se a amitriptilina ou a nortriptilina, que melhoram a continuidade do sono pelo seu forte antagonismo com os receptores histamínicos $\mathrm{H}^{40,41}$. Uma parcela expressiva de pacientes com sono alterado foi avaliada com escalas de sonolência e de qualidade do sono, com melhora substancial das queixas após monoterapia antidepressiva ${ }^{42}$. Contudo, alguns pacientes podem não ter melhora de suas queixas de sono com a monoterapia. Nesses casos, o uso concomitante de hipnóticos indutores do sono (como benzodiazepínicos e anti-histamínicos) e antidepressivos (como amitriptilina ou nortriptilina) pode ser uma opção bem-sucedida ${ }^{21,43}$. Esta abordagem pode, inclusive, acelerar o tratamento da insônia e da SED na depressão, bem como garantir uma maior eficácia na manutenção do sono adequado.

Alguns hipnóticos indutores do sono têm demonstrado uma boa eficácia no tratamento em conjunto com o tratamento antidepressivo. Em um estudo, o uso de zolpidem associado ao manejo antidepressivo melhorou a duração e a qualidade do sono, a habilidade de atenção e de concentração e diminuiu a sonolência diurna ${ }^{44,45}$. Recentemente, o modafinil foi utilizado eficazmente em associação com outros antidepressivos no tratamento da sonolência diurna e depressão, com eliminação da SED e da fadiga. Considerando isso, o modafinil pode atuar com sucesso como medicação coadjuvante a outros tipos de antidepressivos padrões na melhora da condição de sonolência diurna ${ }^{46,47}$.

Como terapia adjuvante, cochilos breves (10 a 15 minutos) em horários específicos (por exemplo, às $13 \mathrm{~h}$ ou $15 \mathrm{~h}$ ) têm sido propostos por alguns grupos de pesquisa ${ }^{48}$.

\section{CONCLUSÕES}

A SED é uma queixa associada ao transtorno depressivo que requer uma adequada avaliação clínica independente de ter sido iniciada antes, durante ou após o quadro depressivo propriamente dito ou como efeito residual da depressão prévia ou esquema terapêutico adotado. As relações do sono, dos transtornos do sono e da depressão são numerosas e intrínsecas. Contudo, apesar de a natureza exata dessas relações ainda não estar nitidamente compreendida, a necessidade de tratá-las é fundamental. A escolha do manejo terapêutico mais adequado ao tratamento da sonolência diurna na depressão pode resultar na remissão das queixas de sono alterado, prevenir as recaídas e melhorar a qualidade do sono e o desempenho cognitivo dos pacientes que sofrem desses transtornos. 


\section{REFERÊNCIAS}

1. Cano-Lozano MC, Espinosa-Fernandez L, Miro E, Buela-Casal G. Una revisión de las alteraciones del sueño en la depresión. Rev Neurol. 2003;36(4):366-75.

2. Chellappa S, Araujo J F. Transtornos do sono em pacientes ambulatoriais com depressão. Rev Psiq Clin. 2006;33(5);233-8.

3. Chang PP, Ford DE, Mead LA. Insomnia in young men and subsequent depression. The Johns Hopkins precursors study. Am J Epidemiol 1997; 146:105-14.

4. Ford DE, Cooper-Patrick L. Sleep disturbances and mood disorders: an epidemiologic perspective. Depress Anxiety. 2001; 14:3-6.

5. Ohayon MM. Epidemiology of insomnia: what we know and what we still need to learn. Sleep Med Rev. 2000;6(2):97-111.

6. American Academy of Sleep Medicine. International classification of sleep disorders, second edition: diagnostic and coding manual. Westchester, IL: American Academy of Sleep Medicine, 2005.

7. Sociedade Brasileira de Sono. I Consenso Brasileiro sobre Insônia. Hypnos. 2003; 4: 3-45.

8. Gupta RM. Approach to the sleepy patient. Medicine and Health. 2002;85(3):86-9.

9. Chellappa SL, Araújo JF. Excessive daytime sleepiness in patients with depressive disorder. Rev Bras Psiquiatr. 2006;28(2):126-30.

10. Bittencourt LR, Silva RS, Santos RF, Pires ML, Mello MT. Excessive daytime sleepiness. Rev Bras Psiquiatr. 2005;27(1): 16-21.

11. National Institutes of Health State of The Science State Conference. Sleep. 2005;28: 1049-57.

12. Lucchesi LM, Pradella-Hallinan M, Lucchesi M, Moraes WA. O sono em transtornos psiquiátricos. Rev Bras Psiquiatr. 2005;27(1):27-32.

13. Hublin C, Kaprio J, Partinen M, Koskenvou M. Insuffient sleep: a population-based study in adults. Sleep. 2001;24(4):392-9.

14. Kräuchi K, Cajochen C, Wirz-Justice A. Thermophysiologic aspects of the three-process-model of sleepiness regulation. Clin Sports Med. 2005;24:287-300.

15. Kräuchi K, Cajochen C, Wirz-J ustice A. Waking up properly: is there a role of thermoregulation in sleep inertia? J Sleep Res. 2004;13:121-7.

16. Wirz-J ustice A. Circadian disturbances in depression: therapeutic perspectives. Medicographia. 2003;25:29-36.

17. Wirz-J ustice A, Van den Hoofdakker RH. Sleep deprivation in depression: what do we know, where do we go? Biol Psychiatry. 1999; 46:445-53.

18. Moore RY. Circadian rhythms: basic neurobiology and clinical applications. Ann Rev Med. 1997; 48: 253-66.

19. Dijk DJ, Duffy JF, Riel E, Shanahan TL, Czeisler CA. Ageing and the circadian and homeostatic regulation of human sleep during forced desynchrony of rest, melatonin and temperature rhythms. J Physiol. 1999;516:611-27.

20. Riemann D, Berger M, Voderholzer U. Sleep and depression - results from psychobiological studies: an overview. Biol Psychology. 2001;57:67-103.

21. Fava M. Daytime sleepiness and insomnia as correlates of depression. J Clin Psychiatry. 2004;65(16):27-32. 
22. Tylee A, Gastpar M, Lepine JP, Mendlewicz J. DEPRESS II (Depression Research in European Society II): a patient survey of the symptoms, disability and current management of depression in the community. DEPRESS Steerling Committee. Int Clin Psychopharmacol. 1999; 14:139-51.

23. Hara C, Lopes Rocha F, Lima-Costa MF. Prevalence of excessive daytime sleepiness and associated factors in a Brazilian community: the Bambui study. Sleep Med. 2004;5:31-6.

24. Voderholzer U, Al-Shajlawi A, Weske G, Feige B, Riemann D. Are there gender differences in objective and subjective sleep measures? A study of insomniacs and healthy controls. Depress Anxiety. 2003; 17: 162-72.

25. Hublin C, Kapiro J, Partinen M, Heikkilä K, Koskenvuo M. Daytime sleepiness in an adult Finnish population. J Intern Med. 1996; 239:417-23.

26. Ohayon MM, Caulet M, Philip P. How sleep and mental disorders are related to daytime sleepiness. Arch Intern Med. 1997; 157:2645-52.

27. Lundt L. Use of the Epwoth Sleepiness Scale to evaluate the symptom of excessive sleepiness in major depressive disorder. Gen Hosp Psychiatry. 2005; 27: 146-8.

28. Bonnet MH, Arand DL. We are chronically sleep deprived. Sleep. 1995; 18:908-11.

29. Young TB. Epidemiology of daytime sleepiness: definitions, symptomatology and prevalence. J Clin Psychiatry. 2004;65(16):12-6.

30. Hoddes E, Zarcone V, Smythe H, Phillips R, Dement WC. Quantification of sleepiness: a new approach. Psychophysiology. 1973;10(4):431-6.

31. Akerstedt T, Gillberg M. Subjective and objective sleepiness in the active individual. Int J Neurosci. 1990;52(1-2):29-37.

32. Johns MW. A new method for measuring daytime sleepiness: the Epworth Sleepiness Scale. Sleep. 1991; 14(6): 540-5.

33. Aldrich MS. Polysomnographic assessment of insomnia. Sleep. 1990;13:188-9.

34. Blatter K, Cajochen C. Circadian rhythms in cognitive performance: Methodological constraints, protocols, theoretical underpinnings. Physiol Behav. 2007; 90(2-3): 196-208.

35. Hudson J L, Pope HG, Sullivan LE, Watermaux CM, Keck PE, Broughton RJ. Good sleep, bad sleep: a meta-analysis of polysomnographic measures of insomnia, depression and narcolepsy. Biol Psychiatry. 1992; 32:958-75.

36. Togeiro SM, Smith AK. Diagnostics methods for sleep disorders. Rev Bras Psiquiatr. 2005;28(1):8-15.

37. American Academy of Sleep Medicine. Sleep related breathing disorders in adults: recommendations for syndrome definitions and measurement techniques in clinical research. The report of an American Academy of Sleep Medicine Task Force. Sleep. 1999;22(5):667-89.

38. Chesson Jr AL, Wise M, Davila D, Johnson S, Littner M, Anderson WM, et al. Practice parameters for the treatment of restless legs syndrome and periodic limb movement disorder. An American Academy of Sleep Medicine Report. Standards of Practice Committee of the American Academy of Sleep Medicine. Sleep. 1999;22(7):961-8.

39. Qureshi A, Lee-Chiong Jr T. Medications and their effects on sleep. Med Clin North Am. 2004;88(3): 751-66.

40. Ohayon MM, Caulet M. Insomnia and psychotropic drug consumption. Prog Neuropsychopharmacol Biol Psychiatry. 1995; 19(3):421-31.

41. Ohayon MM, Caulet M, Priest RG, Guilleminault C. Psychotropic medication consumption 
patterns in the UK general population. J Clin Epidemiol. 1998; 51(3):273-83.

42. Simon GE, Heiligesnstein JH, Grothaus L. Should anxiety and insomnia influence antidepressant selection: a randomized comparison of fluoxetine and imipramine. J Clin Psychiatry. 1998;59:49-55.

43. Benca R. Consequences of Insomnia and its Therapies. J Clin Psychiatry. 2001;62(10):33-7.

44. Perlis ML, McCall WV, Krystal AD, Walsh JK. Long-term, non-nightly administration of zolpidem in the treatment of patients with primary insomnia. J Clin Psychiatry. 2004;65(8):1128-37.

45. Asnis GM, Chakraburtty A, DuBoff EA. Zolpidem for persistent insomnia in SSRI-treated depressed patients. J Clin Psychiatry. 1999;60:668-76.

46. Menza MA, Kaufman KR, Castellanos A. Modafinil augmentation of antidepressant treatment in depression. J Clin Psychiatry. 2000;61:378-81.

47. DeBatista C, Lambke A, Solvason HB. A prospective trial of modafinil as an adjunctive treatment of major depression. J Clin Psychopharm. 2004;24:87-90.

48. Silber MH. Sleep Disorders. Neurol Clin. 2001;19:173-86.

\section{全 Correspondência}

Sarah Laxhmi Chellappa

Wilhelm Kleinstrasse 27, CH-4025, Basel, Switzerland

E-mail: sarahlc_3@hotmail.com

Recebido em 11/06/2007.

Aceito em 11/09/2007.

Não foram declarados conflitos de interesse associados à publicação deste artigo. 